\title{
Review Article \\ Right Ventricle Remodeling and Function in Scleroderma Patients
}

\author{
Roxana Cucuruzac, Iolanda Muntean (D), Imre Benedek, Andras Mester, Nora Rat, \\ Adriana Mitre, Monica Chitu, and Theodora Benedek
}

University of Medicine and Pharmacy of Târgu Mureş, Gh. Marinescu Street, No. 38, 540139 Tîrgu Mureş, Romania

Correspondence should be addressed to Iolanda Muntean; iolanda.muntean@gmail.com

Received 1 September 2017; Accepted 12 February 2018; Published 20 March 2018

Academic Editor: Shigeru Kotake

Copyright (C) 2018 Roxana Cucuruzac et al. This is an open access article distributed under the Creative Commons Attribution License, which permits unrestricted use, distribution, and reproduction in any medium, provided the original work is properly cited.

\begin{abstract}
Scleroderma, known also as systemic sclerosis (SSc), is a severe disease associated with high mortality rates, and right ventricular (RV) remodeling and dysfunction, along with pulmonary artery hypertension (PAH), are among the most important internal organ manifestations of this disease. PAH has a higher prevalence in patients with SSc compared to the general population and represents a significant predictor of mortality in SSc. In patients with SSc, the morphological remodeling and alteration of RV function begin even before the setting of PAH and lead to development of a specific adaptive pattern of the RV which is different from the one recorded in patients with IAPH. These alterations cause worse outcomes and increased mortality rates in SSc patients. Early detection of RV dysfunction and remodeling is possible using modern imaging tools currently available and can indicate the initiation of specific therapeutic measures before installation of PAH. The aim of this review is to summarize the current knowledge related to mechanisms involved in the remodeling and functional alteration of the RV in SSc patients.
\end{abstract}

\section{Introduction}

Scleroderma, known also as systemic sclerosis (SSc), is a severe disease associated with high mortality rates, and right ventricular (RV) remodeling and dysfunction, along with pulmonary artery hypertension (PAH), are among the most important internal organ manifestations of this disease [1].

The mortality of SSc depends primary on the burden of the internal organ involvement. Pulmonary hypertension $(\mathrm{PH})$, pulmonary fibrosis, and kidney damage are the most frequent causes of death in SSc [2,3]. Myocardial, pericardial, and pulmonary artery (PA) involvement is observed in 1 out of 4 patients and serve as predictors of mortality in SSc, as approximately $30 \%$ of the SSc deaths are attributed to cardiac causes, followed by respiratory causes (17\%) [4].

SSc is a complex autoimmune disease characterized by a marked chronic inflammation, which leads to increased connective tissue fibrosis and vascular involvement causing severe damage of internal organs (mainly the heart, lungs, gastrointestinal tract, kidneys, and muscles) and the skin [5].

Activation of the immune system, linked with fibroblast dysfunction, T lymphocyte, macrophage, and mast cell disturbances induce the alteration of the extracellular matrix by secretion of cytokines, chemokines, growth factors, and other potent mediators resulting in excessive collagen deposit in the tissues [6]. The exact etiology and trigger of the disease still remain unknown, but many studies suggest that genetic and environmental factors (silica, solvent, or radiation exposure) play an important role in the pathophysiology of SSc, generating changes in the expression of deoxyribonucleic acid (DNA) and microribonucleic acid (miRNA), which ultimately lead to inadequate activation of the immune system with phenotypic transformation of various cell types $[7,8]$. Infiltration of the microvasculature accompanied by endothelial dysfunction and fibrosis initiates platelet activation and thrombosis causing ischemia of the surrounding tissues $[9,10]$. It has been suggested that eNOS G894T polymorphism is correlated with an increased risk of SSc and PAH as well $[11,12]$. 
The various clinical form of SSc varies from limited cutaneous systemic sclerosis (lcSSc) or CREST (calcinosis, Raynaud phenomenon, esophageal dysmotility, sclerodactyly, and telangiectasias) syndrome, to diffuse, sometimes fulminant forms with extended involvement of internal organs. The incidence of SSc greatly varies between different geographical regions and ethnic groups, with higher numbers among the African American population [13, 14]. The risk of developing SSc is significantly higher in woman than in men, with a $3-6: 1$ female to male ratio [15].

The aim of this review is to summarize the current knowledge related to mechanisms involved in the remodeling and functional alteration of the RV in SSc patients.

\section{Systemic Sclerosis-Related Pulmonary Artery Hypertension (SSc-PAH)}

PAH has a higher prevalence in patients with SSc compared to the general population, being more commonly observed in patients with lcSSc form, and represents a significant predictor of mortality in SSc $[16,17]$. Current literature data suggests that the prevalence of $\mathrm{PAH}$ in this patient category reaches $12 \%$ and, despite the introduction of modern optimized treatments, the 3-year survival rate is still 52\%, compared to $94 \%$ for SSc patients without PAH $[18,19]$. At the same time one-year mortality rate of patients with SSc-PAH is around $30 \%$ versus $15 \%$ in patients with IPAH $[20,21]$. Despite the fact that hemodynamic changes are less pronounced in SScPAH compared to idiopathic PAH (IPAH), SSc-related PAH is associated with twofold to threefold higher mortality risk, due to the systemic nature and complexity of the disease, added to a limited efficiency of the administered therapy $[22,23]$.

Argula et al. evaluated the functional changes of the RV during treatment in patients with SSc-PAH and IPAH at 3.8 and 1.95 years of follow-up. While the IPAH group showed significant improvement of the tricuspid annular plane systolic excursion (TAPSE $[p=0.01]$ ), no such gain was observed in SSc-PAH patients, who also exhibited a worsening trend in tricuspid regurgitation jet velocity, right atrium (RA), and ventricular size [24].

In a comparative study Tedford et al. evidenced worse RV systolic function and prognosis in patients with SSc$\mathrm{PAH}$, compared to IPAH patients at similar RV afterload settings, suggesting the presence of an intrinsic systolic dysfunction in SSc patients [1]. The increase of the pulmonary artery pressure is a result of the alteration of the pulmonary microvascular bed triggered by the chronic inflammation, vasoconstriction, endothelial dysfunction, microthromboses, hypoxia, and excessive fibrous deposits at the level of the arterioles, causing increase of the pulmonary resistance $[25$, 26].

Hassoun et al. performed endomyocardial biopsies of the $\mathrm{RV}$ in SSc patients with and without $\mathrm{PAH}$ and compared these with samples from preserved ejection fraction heart failure (HFpEF) patients. Unpublished data from this study showed a significant decrease of the capillary density in SSc-PAH samples compared to the HFpEF and SSc without
PAH samples. Furthermore, myocardial capillary density was significantly lower in samples from SSc patients with increased right atrial pressure (RAP), compared with lower RAP SSc samples, supporting the theory of structural RV alteration in these patients [27].

\section{Right Ventricular Involvement in Scleroderma: A Distinctive Pattern}

\subsection{Right Ventricular Involvement in Scleroderma Patients} without PAH. Cardiac manifestation of SSc includes involvement of the myocardium, pericardium, and the electrical conduction system, which may lead to ischemia, heart failure, pericardial effusion, and arrhythmias. RV failure can represent an important cause of mortality in SSc patients. It was believed that RV involvement is mainly linked to $\mathrm{PAH}$, but recent studies suggest that SSc may have a direct impact on the RV structure and function, as alteration of RV function is more expressed in SSc-associated PAH than in non-SSc-PAH $[28,29]$. The extent of cardiac involvement is likely to be underestimated, as autopsy studies identified considerable fibrotic changes of the myocardium in $70 \%$ of the examined patients [30]. Focal recurrent ischemia because of microvascular thrombosis, vasospasm thickening of the vascular wall, and fibrous deposits lead to irreversible functional and structural modifications of the myocardium [31, 32]. It is hard to differentiate the primary heart involvement from secondary development of these alterations due to $\mathrm{PAH}$ and kidney injuries. These changes may remain silent for long time (thus frequently underdiagnosed), but when clinically manifested it is described to represent an important negative prognostic factor [33].

Several studies tried to characterize RV involvement that occurs in SSc patients before development of PAH. Proper echocardiographic assessment of the RV function and volume estimation has been bound by its crescent shape, presence of intense trabeculations, and different contraction pattern, but novel techniques overcame these limitations [34]. Pigatto et al. evaluated the RV function of $45 \mathrm{SSc}$ patients without any signs or symptoms of heart disease of $\mathrm{PAH}$ using three-dimensional echocardiography (3DE) and twodimensional speckle-tracking echocardiography (2DSTE) and compared these findings with the similar parameters of 43 healthy subjects. A significant increase in RV size was observed in SSc patients with higher end-systolic volume [ESV, $(p<0.0001)$ ], end-diastolic volume [EDV, $(p=$ $0.049)]$, and reduced ejection fraction $(p<0.0001)$ determined by 3DE. Doppler measurements showed increased systolic pulmonary artery pressure (sPAP) and pulmonary vascular resistance (tPVR) in SSc patients compared to the control group. These changes were more pronounced in patients with lcSSc form [35].

Durmus et al. used 2DSTE for the assessment of RV function in patients with SSc without PAH. Significantly higher sPAP (within normal limits) was recorded in SSc patients ( $p=0.002$ ), accompanied by decreased RV systolic function with significantly lower TAPSE and tissue Doppler maximum systolic myocardial velocity $\left(\mathrm{RVS}^{\prime}\right)$, but no correlation was 


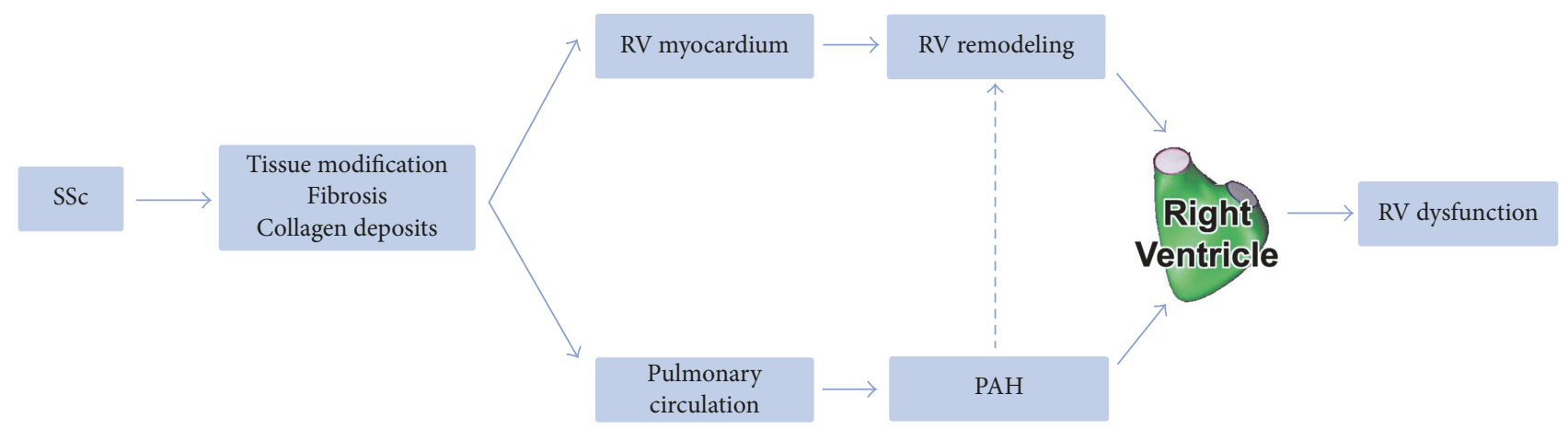

FIGURE 1: The complex interrelation between the mechanisms involved in development of RV dysfunction and remodeling.

observed between these parameters. Right ventricle global longitudinal strain (RVGLS) was also significantly lower in SSc patients compared to the control group $(p<0.001)$. An inverse correlation was observed between duration of the disease and TAPSE and RVGLS [36]. These findings consolidate the earlier results published by Schattke et al. who reported significantly decreased TAPSE and RVS' determined by 2DSTE in SSc patients without PAH and identified isovolumetric acceleration (IVA) as the best predictor of early RV systolic impairment in this patient category [37].

Another early predictor of RV systolic dysfunction in SSc patients determined by 2DSTE may be represented by elevated longitudinal strain rates of the RV, which may serve as an adaptive response to even subtle elevation of the SPAP [38]. Furthermore these early systolic dysfunctions of the RV and RA may presage the sPAP elevation; thus it is important to detect them for the optimization of the further treatment of SSc patients, as recent trials show support for an early aggressive therapy [39-42].

\subsection{The Distinctive Pattern of RV Remodeling and Function in} Scleroderma Patients. Functional and structural alterations of the RV develop even before the setting of PAH in SSc patients, suggesting a remodeling pattern different from the one usually recorded in IPAH. Poor response to optimal therapy was observed in SSc-PAH, ultimately leading to RV failure and death, generating higher mortality rates compared to IPAH patients $[1,43,44]$. These findings led to the emergence of a novel theory about a different adaptive functional and structural remodeling of the RV in SSc patients. The complex interrelation between the mechanisms involved in development of RV dysfunction and remodeling is summarized in Figure 1. Different clinical studies tried to characterize the clinical condition of patients with SSc with or without PAH and to identify predictors of RV involvement and dysfunction. The main clinical studies and their findings are presented in Table 1.

In an autopsy study Overbeek et al. analyzed the histological samples of the RV from SSc-PAH and IPAH patients and compared them with samples taken from healthy controls. Significantly more inflammatory cells were observed in interstitium of the RV from SSc-PAH patients compared to IPAH patients and controls; however the quantity of fibrosis did not show significant differences between the groups, suggesting that the underlying mechanism of RV dysfunction may have multiple factors [45].

Kelemen et al. assessed the RV remodeling of SSc$\mathrm{PAH}$ and IPAH patients using cardiac magnetic resonance imaging (cMRI). No significant differences were recorded in terms of RV mass, RV-EDV, RV ejection fraction, stroke volume, or TAPSE between the two groups [46]. It has been demonstrated that SSc-PAH patients exhibit significantly less pronounced increase of the RV mass (assessed by right ventricular modeling index (RVMI) and volume mass index (VMI)), in response to increased RV load (assessed by PVR and (mean) mPAP) compared to IPAH patients, evidencing a different adaptive hypertrophy mechanism of these patients [46]. However, the benefits and disadvantages gained from RV hypertrophy in this patient category are still under debate [47]. In a recently published article Ramjug et al. did not confirm the aforementioned findings, as no significant differences of RV mass were recorded between SSc-PAH and IPAH patients at increased RV load. Nevertheless, they found a notable correlation between the VMI and PVR in SSc-PAH through the entire range of PVR, which may be a predictor for survival, as earlier studies suggested [48, 49].

The functional reserve of RV may also play an important role in the adaptive remodeling of the RV in SSc. Hsu et al. assessed RV function and morphology during exercise (or atrial pacing) testing in 15 SSc-PAH and 9 IPAH patients with comparable resting RV parameters. The RV contractility of the IPAH group was significantly increased during exercise, while the SSc-PAH group did not display improved contractility leading to important increase of RV-EDV and RV-ESV. The authors attribute these changes to decreased calcium cycling in the myocytes of SSc-PAH group ( $p=$ 0.03 ). The abnormal RV-PA coupling and diminished forcefrequency responsiveness (FFR) of these patients during exercise, along with reduced contractile and diastolic reserve, plead for the depletion of RV functional reserve of SSc-PAH patients. These results highlight the importance of intrinsic $\mathrm{RV}$ dysfunction, which ultimately leads to RV failure in SScPAH patients [50].

Kovacs et al. determined the pulmonary exercise hemodynamics of SSc patients with a 4-year follow-up period, observing significant increase of mPAP $(p=0.02)$ and PVR 


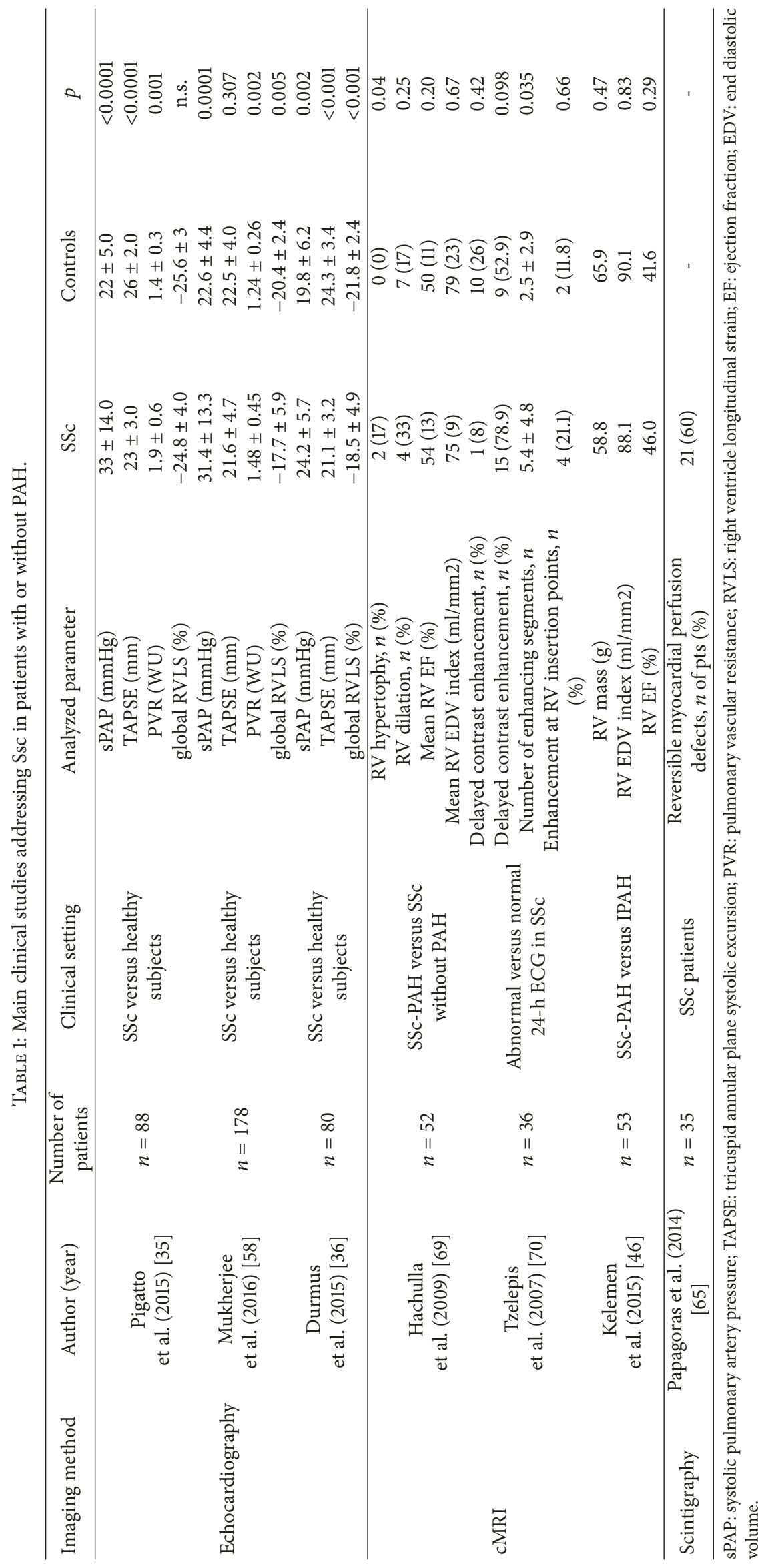


( $p=0.002)$ during exercise, with no changes in resting mPAP values. These findings illustrate the progressive nature of the disease and point out that alterations of exercise hemodynamic parameters precede the routinely determined resting values. The aforementioned changes might be related to the distinctive morphological and functional remodeling pattern of the RV in SSc patients [51].

\section{Imaging Tools for Characterization of the RV in Scleroderma}

Different imaging technologies are currently available for characterization of RF function and morphology, the most used ones being represented by echocardiography, scintigraphy, and cardiac magnetic resonance imaging (cMRI) (Table 1).

4.1. Echocardiography. Given the high prevalence of RV impairment in scleroderma patients, current guidelines recommend early echocardiographic screening of SSc patients at risk for PAH. Immediate initiation of optimal therapy and prevention of right heart failure can improve the prognosis and survival of patients with SSc-PAH [26]. Transthoracic echocardiography (TTE) is a widely available imaging method used for a proper functional and morphological assessment of the RV; however, a considerable number of patients remain undiagnosed until late stages of heart failure, presumably because of the complex geometry of the RV. Conventional 2D echocardiography along with Doppler technology is able to evaluate RV diameters, areas, and volumes, as well as pressures, velocities, and gradients across the cardiac chambers and valves [52].

Different echocardiographic biomarkers have been proposed for characterization of RV function and can serve for evaluation of ventricular dysfunction in patients with SSc. TAPSE is an easily determinable parameter which is considered the most accurate in the assessment of the global RV contractility (albeit it is influenced by load and structure). It correlates with the parameters obtained from right heart catheterization and was proved as a prognostic factor in SSc-PAH $[53,54]$. Another useful parameter for the assessment of RV and global ventricular function is represented by the Tei index, or myocardial performance index (expressed as isovolumetric contraction time and isovolumetric relaxation time divided ejection time), which uses pulsed wave Doppler velocities of ventricular inflow and outflow to calculate ventricular performance. Several studies validated its correlation with lower survival rates in SSc patients [55-57]. Tissue Doppler imaging (TDI) has the advantage to determine myocardial velocities, allowing detection of systolic and diastolic deformations (defined as strain); therefore it is able to assess segmental and global $\mathrm{RV}$ function. The major drawback of this method is the dependence of the Doppler beam angle [34, 39].

Novel echocardiographic modalities based on speckle tracking technology allow a better identification of subclinical heart failure. The technique is a software-based method that allows the calculation of tissue velocity and deformations of the myocardium derived from 2D images, tracking pixels (speckles) of a certain myocardial sector along the cardiac cycle. It can detect the changes in length of the tracked segment during systole and diastole with calculation of strain and strain rate (SR) parameters. These parameters offer an accurate, operator, and angle-independent view of the segmental and global (systolic and diastolic) function of the ventricles. Multiple studies suggest that RV longitudinal strain values and patterns are helpful in the assessment of RV function in SSc and PAH patients and correlate well with the prognosis of this patient category [57-61].

4.2. Scintigraphy. The evaluation of the right ventricular function with nuclear imaging and scintigraphy can determine myocardial damage caused by SSc in early stages by identification of small perfusion defects. A ten-year survival study conducted by Steen et al. showed that SSc patients that presented perfusion defects upon scintigraphy examination with thallium presented a higher rate of cardiac disease and mortality [62]. Furthermore, $99 \mathrm{~m}$ Technetium ventriculography performed on 42 patients without PAH revealed a significantly lower RV ejection fraction compared to controls, both at baseline and at 2 hours from administration of $40 \mathrm{mg}$ of oral Nicardipine [63]. Additionally, patients with SSc may present the so-called "myocardial Raynaud's phenomenon" that leads to transient ischemic episodes induced during the cold pressor test. Lekakis et al. performed dipyridamolethallium-201 scintigraphy during cold pressor testing and found that scleroderma patients presented transient myocardial ischemia induced by cold and that subjects with Raynaud's phenomenon of under 5 years did not present any defects [64]. A combined echocardiography and scintigraphy research study by Papagoras et al. reported that $60 \%$ of patients presented reversible myocardial perfusion defects in spite of the lack of clinically active heart disease, even in younger patients [65].

Although scintigraphy has been proven to be a sensitive method for detection of early perfusion defects in systemic sclerosis patients with no cardiac symptomatology, further studies are needed to elucidate its role in establishing prognosis and therapeutic management, which may be a hard task, since nuclear imaging methods present high costs and are not widely available.

4.3. Cardiac MRI. cMRI is a noninvasive imaging modality that faced a tremendous development in the last decade, being able to provide high quality images on the anatomy and function of the heart [66-68]. cMRI has been shown to offer a significant aid in the diagnosis of several inflammatory processes affecting the myocardium as well as in fibrosis detection and quantification in a great number of pathologies, including SSc [69-73]. cMRI currently is the gold standard method for evaluation of RV parameters, and several studies have focused on the role of cardiac magnetic resonance for assessing right and left ventricular function, the extent and pattern of myocardial fibrosis, and perfusion abnormalities in SSc subjects $[69,70,74]$. Bezante et al. aimed to research the myocardial effects of SSc by cMRI imaging in 50 patients with scarce or no clinical signs of heart failure. The study 
showed that both the right and left ventricular ejection fractions were reduced compared to controls $(p<0.001$ and $p<0.009$, resp.), and the RV ejection fraction matched for body surface area was significantly reduced in subjects with diffuse compared to limited cutaneous SSc [74]. Tzelepis et al. sought to evaluate the distribution and pattern of the fibrotic involvement of the myocardium on 41 patients with SSc with the use of Delayed-Enhancement cMRI (DE-CMR) with gadolinium [70]. Their results revealed that $66 \%$ of the enrolled patients presented late enhancement, predominantly in the middle region of the myocardium wall, with a sparing of the endo- and epicardial regions, predominantly in the middle and basal areas of the left ventricle, with a noncoronary distribution. As for the right ventricular involvement, $17 \%$ of patients presented globular, intermittent enhanced areas in the $\mathrm{RV}$ insertion points, independent of the presence of PAH, upon echocardiographic assessment [70].

Hachulla et al. found that $75 \%$ of SSc patients presented cMRI abnormalities in their study population and that $21 \%$ of patients presented an altered RV ejection fraction, while $17 \%$ showed RV dilation in the absence of PAH. Moreover, they observed the thinning of the left ventricular myocardium that affected primarily patients with diffuse cutaneous SSc and those with no PAH, suggesting that this might be caused by disease's chronic effect on the microvasculature of the heart, being similar to the thinning that occurs during the ventricular remodeling process secondary to infarction [69].

Magnetic resonance imaging of the heart is also a useful tool in evaluating the effects of various therapies in patients with $\mathrm{PAH}$, as well as for prognosis implications. Allanore et al. have evaluated the effect of bosentan on the perfusion and function of the myocardium by performing cMRI and Tissue Doppler Echocardiography on 18 SSc patients with no PA and no symptoms of impaired cardiac function. The study found that cMRI perfusion index and the echocardiography parameters were improved after 4 weeks of bosentan administration [75]. This shows that an improved myocardial function and perfusion can be revealed in SSc patients by highly sensitive imaging methods such as cMRI, which is a valuable noninvasive, nonirradiating, and highly reproducible imaging method useful in this patient population.

\section{Conclusions}

In patients with SSc, the morphological remodeling and alteration of RV function begin even before the setting of $\mathrm{PAH}$ and lead to development of a specific adaptive pattern of the RV which is different from the one recorded in patients with IAPH. These alterations cause worse outcomes and increased mortality rates in SSc patients. Early detection of $\mathrm{RV}$ dysfunction and remodeling is possible using modern imaging tools currently available and can indicate the initiation of specific therapeutic measures before installation of $\mathrm{PAH}$.

\section{Conflicts of Interest}

The authors declare that there are no conflicts of interest regarding the publication of this article.

\section{References}

[1] R. J. Tedford, J. O. Mudd, R. E. Girgis et al., "Right ventricular dysfunction in systemic sclerosis-associated pulmonary arterial hypertension," Circulation: Heart Failure, vol. 6, no. 5, pp. 953963, 2013.

[2] L. Chung, H. W. Farber, R. Benza et al., "Unique Predictors of Mortality in Patients With Pulmonary Arterial Hypertension Associated With Systemic Sclerosis in the REVEAL Registry," CHEST, vol. 146, no. 6, pp. 1494-1504, 2014.

[3] T. A. Winstone, D. Assayag, P. G. Wilcox et al., "Predictors of mortality and progression in scleroderma-associated interstitial lung disease: A systematic review," CHEST, vol. 146, no. 2, pp. 422-436, 2014.

[4] M. Elhai, C. Meune, M. Boubaya et al., "Mapping and predicting mortality from systemic sclerosis," Annals of the Rheumatic Diseases, 2017.

[5] K. Romanowska-Próchnicka, M. Walczyk, and M. Olesińska, "Recognizing systemic sclerosis: Comparative analysis of various sets of classification criteria," Reumatología Clínica, vol. 54, no. 6, pp. 296-305, 2016.

[6] L. I. Sakkas, "New developments in the pathogenesis of systemic sclerosis," Autoimmunity, vol. 38, no. 2, pp. 113-116, 2005.

[7] S. Barsotti, C. Stagnaro, A. d'Ascanio, and A. Della Rossa, "One year in review 2016: Systemic sclerosis," Clinical and Experimental Rheumatology, vol. 34, pp. 3-13, 2016.

[8] A. J. Gilbane, C. P. Denton, and A. M. Holmes, "Scleroderma pathogenesis: A pivotal role for fibroblasts as effector cells," Arthritis Research \& Therapy, vol. 15, no. 3, article no. 215, 2013.

[9] M. Matucci-Cerinic, B. Kahaleh, and F. M. Wigley, "Review: evidence that systemic sclerosis is a vascular disease," Arthritis \& Rheumatology, vol. 65, no. 8, pp. 1953-1962, 2013.

[10] S. A. Jimenez, "Role of endothelial to mesenchymal transition in the pathogenesis of the vascular alterations in systemic sclerosis," ISRN Rheumatology, vol. 2013, Article ID 835948, 15 pages, 2013.

[11] C. Fatini, F. Gensini, E. Sticchi et al., "High prevalence of polymorphisms of angiotensin-converting enzyme (I/D) and endothelial nitric oxide synthase (Glu298Asp) in patients with systemic sclerosis," American Journal of Medicine, vol. 112, no. 7, pp. 540-544, 2002.

[12] R. Togǎnel, I. Muntean, C. Duicu, A. Fǎgărăşan, L. Gozar, and C. Bănescu, "The role of eNOS and AGT gene polymorphisms in secondary pulmonary arterial hypertension in Romanian children with congenital heart disease," Revista Romana de Medicina de Laborator, vol. 21, no. 3-4, pp. 267-274, 2013.

[13] J. Barnes and M. D. Mayes, "Epidemiology of systemic sclerosis: Incidence, prevalence, survival, risk factors, malignancy, and environmental triggers," Current Opinion in Rheumatology, vol. 24, no. 2, pp. 165-170, 2012.

[14] A. C. Gelber, R. L. Manno, A. A. Shah et al., "Race and association with disease manifestations and mortality in scleroderma : A 20-year experience at the johns hopkins scleroderma center and review of the literature," Medicine (United States), vol. 92, no. 4, pp. 191-205, 2013.

[15] C. Nguyen, A. Bérezné, T. Baubet et al., "Association of gender with clinical expression, quality of life, disability, and depression and anxiety in patients with systemic sclerosis," PLOS ONE, vol. 6, no. 3, Article ID e17551, 2011.

[16] J. Le Pavec, M. Humbert, L. Mouthon, and P. M. Hassoun, "Systemic sclerosis-associated pulmonary arterial hypertension," 
American Journal of Respiratory and Critical Care Medicine, vol. 181, no. 12, pp. 1285-1293, 2010.

[17] C. J. Mullin and S. C. Mathai, "New insights into the recognition, classification and management of systemic sclerosis-associated pulmonary hypertension," Current Opinion in Rheumatology, vol. 29, no. 6, pp. 561-567, 2017.

[18] G. Lefèvre, L. Dauchet, E. Hachulla et al., "Survival and prognostic factors in systemic sclerosis-associated pulmonary hypertension: a systematic review and meta-analysis," Arthritis \& Rheumatology, vol. 65, no. 9, pp. 2412-2423, 2013.

[19] A. Tyndall, "Systemic sclerosis in Europe: first report from the EULAR Scleroderma Trials And Research (EUSTAR) group database," Annals of the Rheumatic Diseases, vol. 64, no. 7, pp. 1107-1107, 2005.

[20] M. Hinchcliff, A. Fischer, E. Schiopu, and V. D. Steen, "Pulmonary hypertension assessment and recognition of outcomes in scleroderma (PHAROS): Baseline characteristics and description of study population," The Journal of Rheumatology, vol. 38, no. 10, pp. 2172-2179, 2011.

[21] G. Simonneau, M. Gatzoulis, and I. Adatia, "Updated clinical classification of pulmonary hypertension," Journal of the American College of Cardiology, vol. 62, no. 25, supplement, 2013.

[22] M. R. Fisher, S. C. Mathai, H. C. Champion et al., "Clinical differences between idiopathic and scleroderma-related pulmonary hypertension," Arthritis \& Rheumatology, vol. 54, no. 9, pp. 3043-3050, 2006.

[23] A. Campo, S. C. Mathai, J. Le Pavec et al., "Hemodynamic predictors of survival in scleroderma-related pulmonary arterial hypertension," American Journal of Respiratory and Critical Care Medicine, vol. 182, no. 2, pp. 252-260, 2010.

[24] R. G. Argula, A. Karwa, A. Lauer et al., "Differences in right ventricular functional changes during treatment between systemic sclerosis-associated pulmonary arterial hypertension and idiopathic pulmonary arterial hypertension," Annals of the American Thoracic Society, vol. 14, no. 5, pp. 682-689, 2017.

[25] V. McLaughlin, R. N. Channick, H.-A. Ghofrani et al., "Bosentan added to sildenafil therapy in patients with pulmonary arterial hypertension," European Respiratory Journal, vol. 46, no. 2, pp. 405-413, 2015.

[26] N. Galiè, M. Humbert, J. L. Vachiery et al., "2015 ESC/ERS Guidelines for the diagnosis and treatment of pulmonary hypertension: The Joint Task Force for the Diagnosis and Treatment of Pulmonary Hypertension of the European Society of Cardiology (ESC) and the European Respiratory Society (ERS): Endorsed by: Association for European Paediatric and Congenital Cardiology (AEPC), International Society for Heart and Lung Transplantation (ISHLT)," European Heart Journal, vol. 37, no. 1, pp. 67-119, 2016.

[27] P. M. Hassoun, “The right ventricle in scleroderma (2013 Grover conference series)," Pulmonary Circulation, vol. 5, no. 1, pp. 314,2015 .

[28] S. C. Mathai, M. Bueso, L. K. Hummers et al., "Disproportionate elevation of $\mathrm{N}$-terminal pro-brain natriuretic peptide in scleroderma-related pulmonary hypertension," European Respiratory Journal, vol. 35, no. 1, pp. 95-104, 2010.

[29] M. J. Overbeek, J.-W. Lankhaar, N. Westerhof et al., "Right ventricular contractility in systemic sclerosis-associated and idiopathic pulmonary arterial hypertension," European Respiratory Journal, vol. 31, no. 6, pp. 1160-1166, 2008.
[30] W. P. Follansbee, T. R. Miller, E. I. Curtiss et al., "A controlled clinicopathologic study of myocardial fibrosis in systemic sclerosis (scleroderma)," The Journal of Rheumatology, vol. 17, no. 5, pp. 656-662, 1990.

[31] C. Meune, O. Vignaux, A. Kahan, and Y. Allanore, "Heart involvement in systemic sclerosis: evolving concept and diagnostic methodologies," Archives of Cardiovascular Diseases, vol. 103, no. 1, pp. 46-52, 2010.

[32] F. Fernandes, F. J. A. Ramires, E. Arteaga, B. M. Ianni, E. S. D. O. Bonfá, and C. Mady, "Cardiac remodeling in patients with systemic sclerosis with no signs or symptoms of heart failure: An endomyocardial biopsy study," Journal of Cardiac Failure, vol. 9, no. 4, pp. 311-317, 2003.

[33] S. Lambova, "Cardiac manifestations in systemic sclerosis," World Journal of Cardiology, vol. 6, no. 9, p. 993, 2014.

[34] J. Kjærgaard, "Assessment of right ventricular systolic function by tissue Doppler echocardiography.", Danish Medical Journal, vol. 59, no. 3, p. B4409, 2012.

[35] E. Pigatto, D. Peluso, E. Zanatta et al., "Evaluation of right ventricular function performed by 3d-echocardiography in scleroderma patients," Reumatismo, vol. 66, no. 4, pp. 259-263, 2015.

[36] E. Durmus, M. Sunbul, K. Tigen et al., "Right ventricular and atrial functions in systemic sclerosis patients without pulmonary hypertension: Speckle-tracking echocardiographic study," Herz, vol. 40, no. 4, pp. 709-715, 2015.

[37] S. Schattke, F. Knebel, A. Grohmann et al., "Early right ventricular systolic dysfunction in patients with systemic sclerosis without pulmonary hypertension: A Doppler Tissue and Speckle Tracking echocardiography study," Cardiovascular Ultrasound, vol. 8, no. 1, article no. 3, 2010.

[38] C. Matias, L. P. D. Isla, M. Vasconcelos et al., "Speckle-trackingderived strain and strain-rate analysis: A technique for the evaluation of early alterations in right ventricle systolic function in patients with systemic sclerosis and normal pulmonary artery pressure," Journal of Cardiovascular Medicine, vol. 10, no. 2, pp. 129-134, 2009.

[39] M. D’Alto, G. Cuomo, E. Romeo et al., “Tissue Doppler imaging in systemic sclerosis: A 3-year longitudinal study, Seminars in Arthritis and Rheumatism, vol. 43, no. 5, pp. 673-680, 2014.

[40] P. M. Hassoun, R. T. Zamanian, R. Damico et al., "Ambrisentan and tadalafil up-front combination therapy in sclerodermaassociated pulmonary arterial hypertension," American Journal of Respiratory and Critical Care Medicine, vol. 192, no. 9, pp. 1102-1110, 2015.

[41] G. Kovacs and H. Olschewski, "Borderline pulmonary pressures in scleroderma-a pre-pulmonary arterial hypertension condition?" Arthritis Research \& Therapy, vol. 17, no. 1, p. 123, 2015.

[42] A. D’Andrea, M. D’Alto, M. Di Maio et al., "Right atrial morphology and function in patients with systemic sclerosis compared to healthy controls: a two-dimensional strain study," Clinical Rheumatology, vol. 35, no. 7, pp. 1733-1742, 2016.

[43] M. Rubenfire, M. D. Huffman, S. Krishnan, J. R. Seibold, E. Schiopu, and V. V. McLaughlin, "Survival in systemic sclerosis with pulmonary arterial hypertension has not improved in the modern era," CHEST, vol. 144, no. 4, pp. 1282-1290, 2013.

[44] M. R. Fisher, S. C. Mathai, H. C. Champion et al., "Clinical differences between idiopathic and scleroderma-related pulmonary hypertension," Arthritis \& Rheumatology, vol. 54, no. 9, pp. 3043-3050, 2006.

[45] M. J. Overbeek, K. T. B. Mouchaers, H. M. Niessen et al., "Characteristics of interstitial fibrosis and inflammatory cell 
infiltration in right ventricles of systemic sclerosis-associated pulmonary arterial hypertension," International Journal of Rheumatology, vol. 2010, Article ID 604615, 2010.

[46] B. W. Kelemen, S. C. Mathai, R. J. Tedford et al., "Right ventricular remodeling in idiopathic and scleroderma-associated pulmonary arterial hypertension: Two distinct phenotypes," Pulmonary Circulation, vol. 5, no. 2, pp. 327-334, 2015.

[47] A. Harrison, N. Hatton, and J. J. Ryan, "The right ventricle under pressure: Evaluating the adaptive and maladaptive changes in the right ventricle in pulmonary arterial hypertension using echocardiography (2013 Grover conference series)," Pulmonary Circulation, vol. 5, no. 1, pp. 29-47, 2015.

[48] S. Ramjug, N. Hussain, J. Hurdman et al., "Idiopathic and Systemic Sclerosis-Associated Pulmonary Arterial Hypertension: A Comparison of Demographic, Hemodynamic, and MRI Characteristics and Outcomes," CHEST, vol. 152, no. 1, pp. 92102, 2017.

[49] D. Hagger, R. Condliffe, N. Woodhouse et al., "Ventricular mass index correlates with pulmonary artery pressure and predicts survival in suspected systemic sclerosis-associated pulmonary arterial hypertension," Rheumatology, vol. 48, no. 9, pp. 11371142, 2009.

[50] S. Hsu, B. A. Houston, E. Tampakakis et al., "Right ventricular functional reserve in pulmonary arterial hypertension," Circulation, vol. 133, no. 24, pp. 2413-2422, 2016.

[51] G. Kovacs, A. Avian, N. Wutte et al., "Changes in pulmonary exercise haemodynamics in scleroderma: A 4-year prospective study," European Respiratory Journal, vol. 50, no. 1, Article ID 1601708, 2017.

[52] E. Hachulla, D. Launay, A. Yaici et al., "Pulmonary arterial hypertension associated with systemic sclerosis in patients with functional class II dyspnoea: mild symptoms but severe outcome.," Rheumatology, vol. 49, no. 5, pp. 940-944, 2010.

[53] P. R. Forfia, M. R. Fisher, S. C. Mathai et al., "Tricuspid annular displacement predicts survival in pulmonary hypertension," American Journal of Respiratory and Critical Care Medicine, vol. 174, no. 9, pp. 1034-1041, 2006.

[54] S. C. Mathai, C. T. Sibley, P. R. Forfia et al., "Tricuspid annular plane systolic excursion is a robust outcome measure in systemic sclerosis-associated pulmonary arterial hypertension," The Journal of Rheumatology, vol. 38, no. 11, pp. 2410-2418, 2011.

[55] C. Tei, "New non-invasive index for combined systolic and diastolic ventricular function," Journal of Cardiology, vol. 26, no. 2, pp. 135-136, 1995.

[56] S. Frea, M. Capriolo, W. G. Marra et al., "Echo doppler predictors of pulmonary artery hypertension in patients with systemic sclerosis," Journal of Echocardiography, vol. 28, no. 8, pp. 860-869, 2011.

[57] H. Blessberger and T. Binder, "Two dimensional speckle tracking echocardiography: Basic principles," Heart, vol. 96, no. 9, pp. 716-722, 2010.

[58] M. Mukherjee, S. Chung, V. K. Ton et al., "Unique abnormalities in right ventricular longitudinal strain in systemic sclerosis patients," Circulation: Cardiovascular Imaging, vol. 9, article e003792, no. 6, 2016.

[59] A. Meris, F. Faletra, C. Conca et al., "Timing and magnitude of regional right ventricular function: A speckle trackingderived strain study of normal subjects and patients with right ventricular dysfunction," Journal of the American Society of Echocardiography, vol. 23, no. 8, pp. 823-831, 2010.

[60] L. G. Rudski, W. W. Lai, J. Afilalo et al., "Guidelines for the echocardiographic assessment of the right heart in adults: a report from the American Society of Echocardiography endorsed by the European Association of Echocardiography, a registered branch of the European Society of Cardiology, and the Canadian Society of Echocardiography," Journal of the American Society of Echocardiography, vol. 23, no. 7, pp. 685-713, 2010.

[61] I. Muntean, T. Benedek, M. Melinte, C. Suteu, and R. Togãnel, "Deformation pattern and predictive value of right ventricular longitudinal strain in children with pulmonary arterial hypertension," Cardiovascular Ultrasound, vol. 14, no. 1, article no. 27, 2016.

[62] V. D. Steen, W. P. Follansbee, C. G. Conte, and T. A. Medsger Jr., "Thallium perfusion defects predict subsequent cardiac dysfunction in patients with systemic sclerosis," Arthritis \& Rheumatology, vol. 39, no. 4, pp. 677-681, 1996.

[63] C. Meune, Y. Allanore, J.-Y. Devaux et al., "High prevalence of right ventricular systolic dysfunction in early systemic sclerosis," The Journal of Rheumatology, vol. 31, no. 10, pp. 19411945, 2004.

[64] J. Lekakis, M. Mavrikakis, and M. Emmanuel, "Cold-induced coronary Raynaud's phenomenon in patients with systemic sclerosis," Clinical and Experimental Rheumatology, vol. 16, no. 2, pp. 135-140, 1998.

[65] C. Papagoras, K. Achenbach, N. Tsifetaki, S. Tsiouris, A. Fotopoulos, and A. A. Drosos, "Heart involvement in systemic sclerosis: A combined echocardiographic and scintigraphic study," Clinical Rheumatology, vol. 33, no. 8, pp. 1105-1111, 2014.

[66] R. C. Hendel, M. R. Patel, C. M. Kramer et al., "ACCF/ACR/ SCCT/SCMR/ASNC/NASCI/SCAI/SIR 2006 appropriateness criteria for cardiac computed tomography and cardiac magnetic resonance imaging: a report of the American College of Cardiology Foundation Quality Strategic Directions Committee Appropriateness Criteria Working Group, American College of Radiology, Society of Cardiovascular Computed Tomography, Society for Cardiovascular Magnetic Resonance, American Society of Nuclear Cardiology, North American Society for Cardiac Imaging, Society for Cardiovascular Angiography and Interventions, and Society of Interventional Radiology," Journal of the American College of Cardiology, vol. 48, pp. 1475-1497, 2006.

[67] J. P. Ridgway, "Cardiovascular magnetic resonance physics for clinicians: Part i," Journal of Cardiovascular Magnetic Resonance, vol. 12, no. 1, article no. 71, 2010.

[68] J. D. Biglands, A. Radjenovic, and J. P. Ridgway, "Cardiovascular magnetic resonance physics for clinicians: Part II," Journal of Cardiovascular Magnetic Resonance, vol. 14, no. 1, article no. 66, 2012.

[69] A.-L. Hachulla, D. Launay, V. Gaxotte et al., "Cardiac magnetic resonance imaging in systemic sclerosis: A cross-sectional observational study of 52 patients," Annals of the Rheumatic Diseases, vol. 68, no. 12, pp. 1878-1884, 2009.

[70] G. E. Tzelepis, N. L. Kelekis, S. C. Plastiras et al., "Pattern and distribution of myocardial fibrosis in systemic sclerosis: A delayed enhanced magnetic resonance imaging study," Arthritis \& Rheumatology, vol. 56, no. 11, pp. 3827-3836, 2007.

[71] O. Vignaux, Y. Allanore, C. Meune et al., "Evaluation of the effect of nifedipine upon myocardial perfusion and contractility using cardiac magnetic resonance imaging and tissue Doppler echocardiography in systemic sclerosis," Annals of the Rheumatic Diseases, vol. 64, no. 9, pp. 1268-1273, 2005.

[72] M. A. Olimulder, J. van Es, and M. A. Galjee, "The importance of cardiac MRI as a diagnostic tool in viral myocarditis-induced 
cardiomyopathy," Netherlands Heart Journal, vol. 17, no. 12, pp. 481-486, 2009.

[73] B. Heydari, M. Jerosch-Herold, and R. Y. Kwong, "Assessment of Myocardial Ischemia with Cardiovascular Magnetic Resonance," Progress in Cardiovascular Diseases, vol. 54, no. 3, pp. 191-203, 2011.

[74] G. P. Bezante, D. Rollando, M. Sessarego et al., "Cardiac magnetic resonance imaging detects subclinical right ventricular impairment in systemic sclerosis," The Journal of Rheumatology, vol. 34, pp. 2431-2437, 2007.

[75] Y. Allanore, C. Meune, O. Vignaux, S. Weber, P. Legmann, and A. Kahan, "Bosentan increases myocardial perfusion and function in systemic sclerosis: A magnetic resonance imaging and Tissue-Doppler echography study," The Journal of Rheumatology, vol. 33, no. 12, pp. 2464-2469, 2006. 


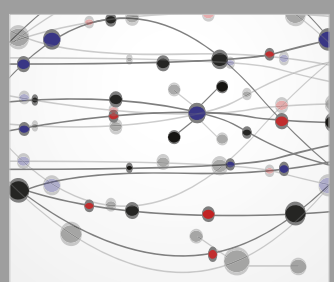

The Scientific World Journal
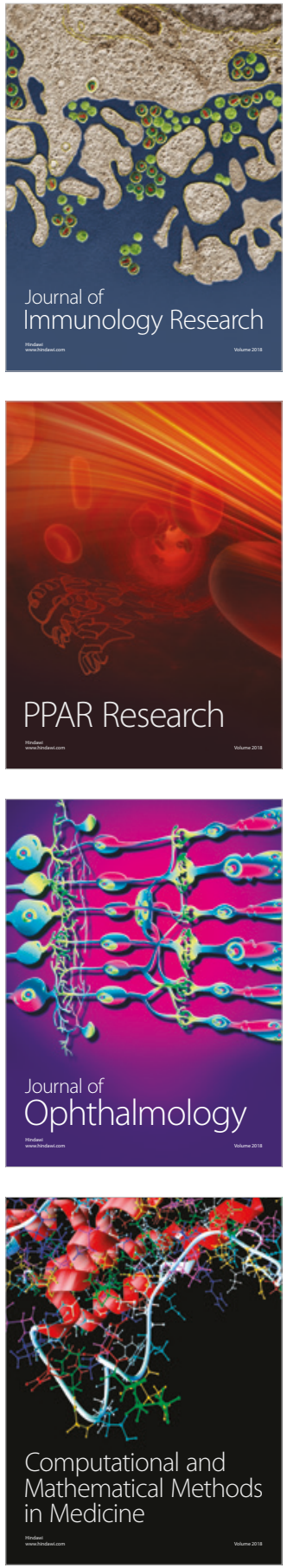

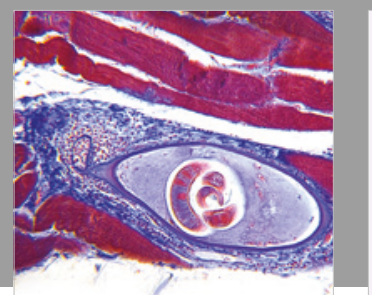

Gastroenterology Research and Practice

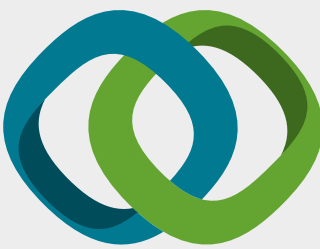

\section{Hindawi}

Submit your manuscripts at

www.hindawi.com
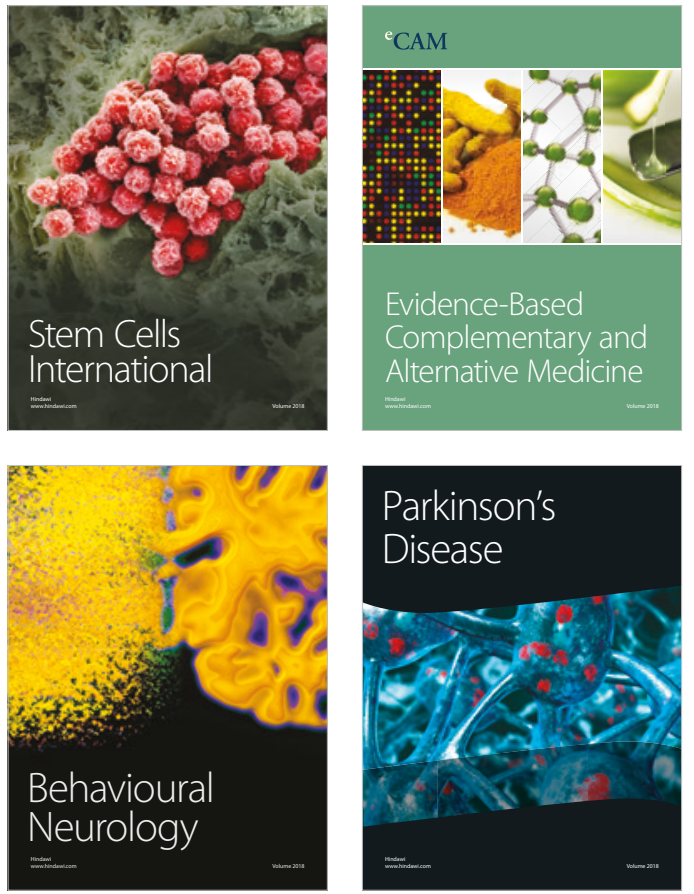

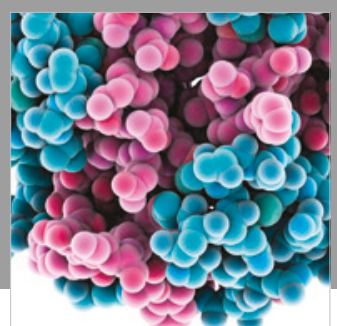

ournal of

Diabetes Research

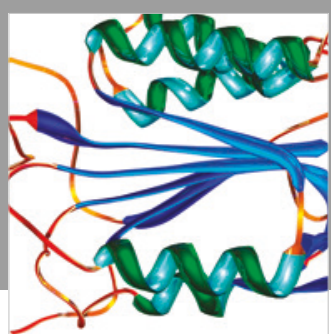

Disease Markers
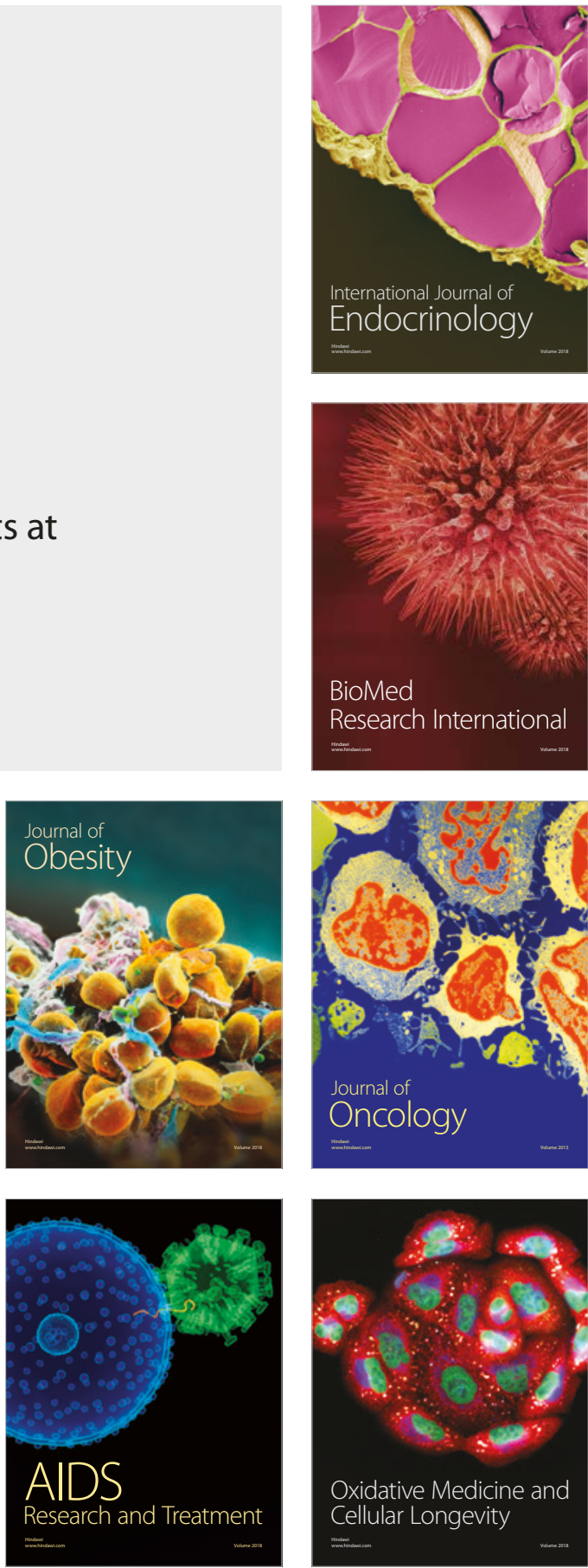